\title{
Delayed Response to Animate Implied Motion in Human Motion Processing Areas
}

\author{
Jeannette A. M. Lorteije ${ }^{1}$, J. Leon Kenemans ${ }^{1}$, Tjeerd Jellema ${ }^{1,2}$, \\ Rob H. J. van der Lubbe ${ }^{1}$, Frederiek de Heer ${ }^{1}$, \\ and Richard J. A. van Wezel ${ }^{1}$
}

\begin{abstract}
Viewing static photographs of objects in motion evokes higher fMRI activation in the human medial temporal complex $(\mathrm{MT}+)$ than looking at similar photographs without this implied motion. As MT + is traditionally thought to be involved in motion perception (and not in form perception), this finding suggests feedback from object-recognition areas onto MT + . To investigate this hypothesis, we recorded extracranial potentials evoked by the sight of photographs of biological agents with and without implied motion. The difference in potential be-
\end{abstract}

\section{INTRODUCTION}

The accurate perception of motion cues is vital for interacting in a dynamic world. The processing of visual object motion in humans involves neuronal activity in specific brain areas, most notably the medio-temporal areas (MT+, also called V5), which are located at the junction of the occipital, parietal, and temporal lobes and are part of the dorsal visual pathway (Ungerleider \& Mishkin, 1982). It is well established that the primary function of the MT+ complex is to analyze the direction and speed of object motion in the visual world, as shown by a plethora of single-cell studies in monkeys (e.g., Britten, Shadlen, Newsome, \& Movshon, 1992) and imaging studies in humans (e.g., Sunaert, Van Hecke, Marchal, \& Orban, 1999; Tootell et al., 1995).

\section{Implied Motion}

In addition to perceiving object motion per se, predicting a moving object's future position is critical for survival, for instance, for evading cars when crossing a busy street, or for aiming at fleeing animals when hunting. Human psychophysical studies have shown that observers extrapolate the remembered position of objects according to the direction of motion implied by form information (Freyd, 1983). Recently, there has been

\footnotetext{
${ }^{1}$ Helmholtz Research Institute, Utrecht University, The Netherlands, ${ }^{2}$ Hull University, United Kingdom
}

tween responses to pictures with and without implied motion was maximal between 260 and $400 \mathrm{msec}$ after stimulus onset. Source analysis of this difference revealed one bilateral, symmetrical dipole pair in the occipital lobe. This area also showed a response to real motion, but approximately $100 \mathrm{msec}$ earlier than the implied motion response. The longer latency of the implied motion response in comparison to the real motion response is consistent with a feedback projection onto MT+ following object recognition in higher-level temporal areas.

growing awareness that the classical motion-sensitive areas do utilize static form information, especially when it implies motion. For example, functional MRI studies have shown a higher BOLD response to photographs of objects that imply motion (e.g., a running athlete or a cup falling from a table), than to photographs without implied motion (e.g., a sitting person or a cup on a table), in the human MT+ complex (hMT+) (Kourtzi \& Kanwisher, 2000; Senior et al., 2000). Furthermore, when coherent firing in this area is disrupted by application of transcranial magnetic stimulation, the mental extrapolation of the location of an object implying motion disappears, indicating the functional necessity of hMT + for implied motion perception (Senior et al., 2002). Additionally, Krekelberg et al. (2003) showed that monkey MT also processes motion implied by the inanimate form information of Glass patterns.

These findings are surprising because form processing is thought to occur along the ventral pathway (Ungerleider \& Mishkin, 1982). It raises the question how (animate) implied motion information arrives in hMT + . If it is via feedback from higher processing centers, then the latency of hMT+ activation should be increased relative to activation by real motion, which typically occurs from 150 to 200 msec after motion onset (Hoffmann et al., 2001; Probst, Plendl, Paulus, Wist, \& Scherg, 1993). To determine the response latency of implied motion and re-establish the response latency of real motion, we used EEG scans with a high temporal resolution to record the visually evoked potential (VEP) to 


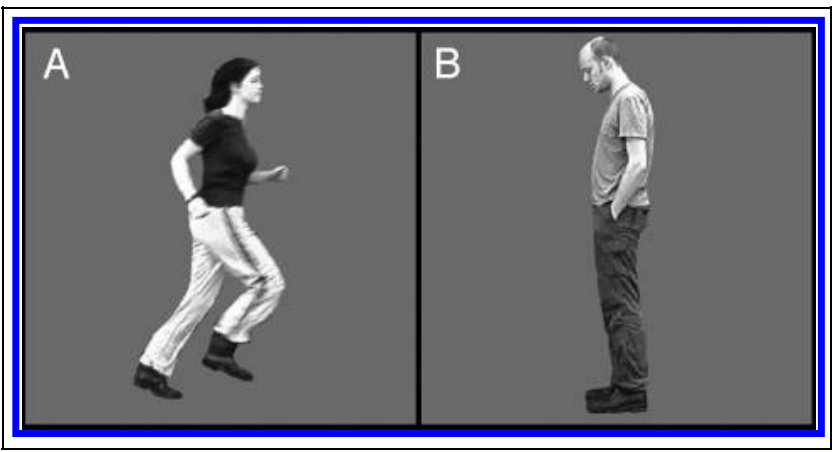

Figure 1. Examples of photographs with implied motion (A) and without implied motion (B) that were used in this study. All photographs were presented at both left and right profiles.

photographs with versus photographs without implied motion (Figure 1), as well as to moving stimuli.

Interestingly, both fMRI studies investigating implied motion (Kourtzi \& Kanwisher, 2000; Senior et al., 2000) found very similar results, even though their paradigms differed at some points, most notably with respect to the moving stimuli used to locate area hMT + . Kourtzi and Kanwisher (2000) used the difference in response to low-contrast moving concentric rings and static rings, whereas Senior et al. (2000) used movie clips of moving objects and the same objects at rest. Although the moving rings are standard stimuli for hMT + activation, the movie clips provide a better comparison to implied motion (the implied motion stimuli were frames taken from these clips). Both methods successfully located area hMT+ in fMRI scans. However, it is not certain what might provide an optimal localization of the motionsensitive cortex in spatially poorer EEG recordings. Therefore, we used in two different experiments either inanimate motion (random dot patterns [RDPs]: moving vs. static or vs. flicker) and animate motion (movie clip of running person vs. one frame of that movie). Additionally, we were interested in the influence of luminance on the evoked potentials. Therefore, we used luminance-uncorrected photographs in the first (inanimate RDP motion) experiment, in contrast to luminance-corrected photographs in the second (animate movie clip) experiment.

\section{RESULTS}

\section{Implied Motion}

\section{Visually Evoked Potentials}

We recorded EEG signals in 14 human subjects that were viewing (luminance uncorrected) photographs of other people in running (implied motion) or stationary (nonimplied motion) posture. Examples of the stimuli are presented in Figure 1, a complete description of the visual stimuli and task is given in the Methods section. Differences between implied versus nonimplied conditions were most pronounced at occipital-parietal electrodes (Figure 2). To statistically test differences between conditions, a general linear model with repeated measures was applied to mean amplitudes across
Figure 2. The implied motion effect is maximal from 260 to $320 \mathrm{msec}$ in the luminanceuncorrected experiment $(n=14)$. This is visible as a large deviation between the grand-average VEPs to implied motion (black line) and nonimplied motion (gray line) at electrode positions PO4 (left) and $\mathrm{Pz}$ (right). The dotted line depicts the difference curve between the implied and nonimplied VEPs, and the dotted bar highlights the periods during which the two differed significantly (general linear model: Condition $\times$ Electrodes, Greenhouse-Geisser $p$ value $<.05)$. From 60 to $100 \mathrm{msec}$, a smaller (but significant) deviation occurred, which might be attributed to a response to low-level stimulus differences, especially luminance.

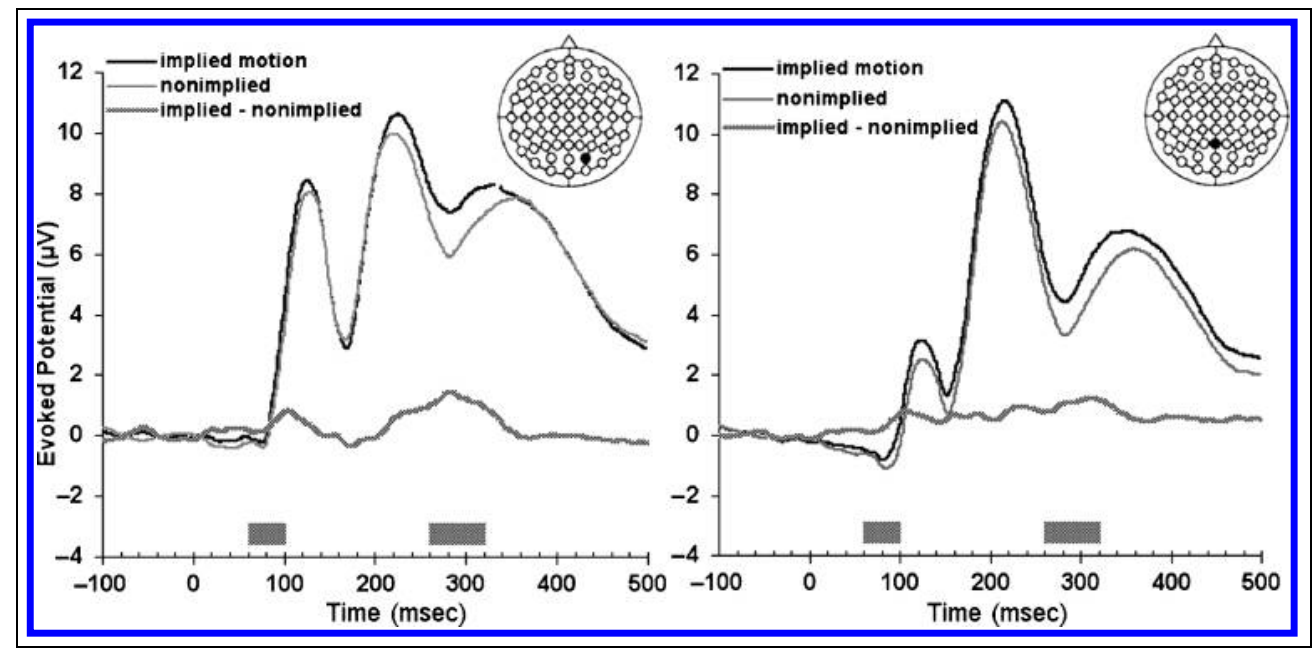


20-msec time segments, with condition (implied and nonimplied) and electrode position as within-subject factors. A significant difference (Greenhouse Geisser $p$ value $<.05)$ in response to photographs with implied motion versus photographs with nonimplied motion was found from 60 to $100 \mathrm{msec}$, and from 260 to 320 msec after stimulus onset.

The first difference was strongest at electrode $\mathrm{Pz}$ during a small negative peak. Evoked potentials during this time interval have been shown to reflect luminance (Johannes, Münte, Heinze, \& Mangun, 1995), spatial frequency (Kenemans, Baas, Mangun, Lijffijt, \& Verbaten, 2000), orientation (Arakawa et al., 2000), and size and eccentricity differences (Busch, Debener, Kranczioch, Engel, \& Herrmann, 2004), which indicate that this early divergence reflected low-level stimulus differences between the two conditions. For 11 subjects, the implied response was more positive than the nonimplied motion response. One subject showed a slightly more negative implied motion response, and for two subjects there was no clear difference between the responses to the two conditions.

The second difference (from 260 to $320 \mathrm{msec}$ ) between the two photograph conditions was much more pronounced, and was clearly visible at most occipital and occipital-parietal electrodes. A general linear model analysis on a subset of 35 electrodes ( 5 lateral $/$ medial by 7 anterior/posterior locations) confirmed a significantly different anterior/posterior gradient between these two conditions. The implied motion photographs evoked a more positive potential than the nonimplied photographs at posterior electrodes, which was clearly noticeable in the grand average and in the individual data of 11 of the 14 subjects. Because activity due to luminance, spatial frequency, orientation, size, and eccentricity differences occur much earlier, this divergence between implied and nonimplied conditions most probably reflected implied motion activity. Subse- quently, we refer to this difference as the implied motion response.

\section{Luminance}

In a second experiment with seven subjects, the average luminance of the implied motion pictures was adjusted to match their nonimplied counterparts. Because the first (luminance uncorrected) experiment already revealed that the differences between the implied and nonimplied responses were maximal at electrode $\mathrm{Pz}$ (early difference) and PO4 (late difference), we tested differences between implied and nonimplied responses at these two electrodes in a paired $t$ test (Figure 3).

Differences at electrode PO4 were significant $(p<.05)$ from 120 to $140 \mathrm{msec}$ and from 300 to $380 \mathrm{msec}$ after stimulus onset. Differences at electrode $\mathrm{Pz}$ were significant from 120 to $160 \mathrm{msec}$, from 220 to $260 \mathrm{msec}$, and from 340 to $400 \mathrm{msec}$. The first difference (120 to $160 \mathrm{msec}$ ) is comparable to the 60 to $100 \mathrm{msec}$ difference in the first experiment, whereas the late difference between 300 and $400 \mathrm{msec}$ is very similar in shape as the 260 to $320 \mathrm{msec}$ difference in the first experiment. As in the first experiment, the first difference was small and difficult to discriminate in comparison to the late response, which was clearly visible as a positive potential in the grand average and in the individual data of five out of seven subjects.

The longer latencies in this second experiment (when compared to the results of the first experiment) cannot be explained by the luminance adjustment, as only the implied motion photographs were adjusted, whereas the nonimplied photographs remained unaltered. Implied and nonimplied responses in this second experiment had the same response latencies. These longer latencies might thus be attributed to group differences.

Electrode $\mathrm{Pz}$ revealed an additional significant difference between implied and nonimplied responses from 220 to 260 msec after stimulus onset. Because this
Figure 3. Grand averages for luminance-corrected photographs $(n=7)$ showed the same deviation as luminance-uncorrected photographs (Figure 2), albeit at a longer latency. The dotted bars indicate the time periods during which significant differences (paired $t$ test $p$ value $<.05$ ) between implied and nonimplied potentials occur at electrodes PO4 (left) and $\mathrm{Pz}$ (right).

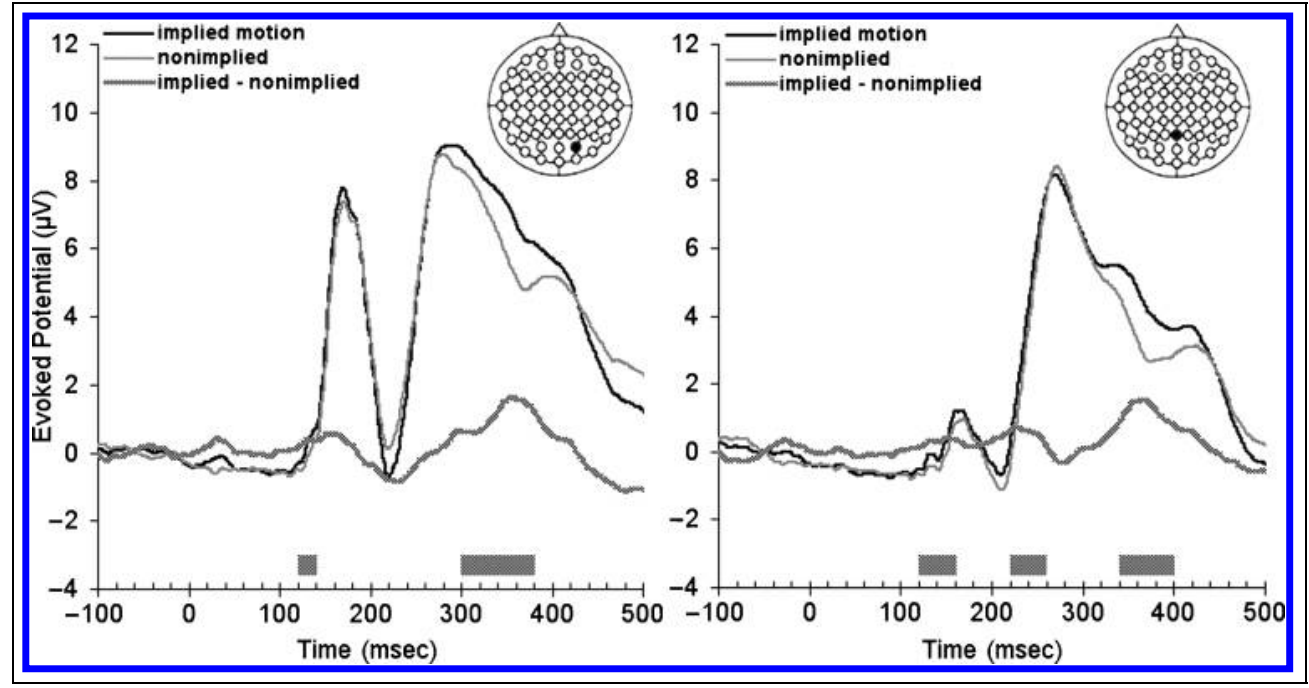


difference did not occur at electrode PO4, and was absent in the first experiment, it was not taken into account during further analysis.

\section{Implied Motion Source Localization}

To estimate the locations of neural sources underlying differences between conditions, dipole modeling (brain electric source analysis [BESA 2.2]: Berg \& Scherg, 1994; Scherg \& Picton, 1991) was performed on the subtraction VEPs: implied motion minus nonimplied photographs. Source models consisted of single dipole pairs, whose bilateral locations and orientations were mirrored in the midline.

Scalp data from the implied minus nonimplied subtraction grand averages were analyzed for those time periods that the VEPs of the two conditions differed significantly. When the global field power was maximal during these periods, a 3-msec timeframe was chosen for source localization. The difference in response between the two photograph conditions from 60 to 100 msec could not be fitted by a symmetrical dipole model that would fit the grand average, due to its low global field power and large variance over participants. Therefore, sources were located per individual at the moment of the highest (individual) global field power within 60 to $100 \mathrm{msec}$ after stimulus onset. Individual locations varied across participants (Figure 4). Residual variances (RVs, the percentage of scalp data that the

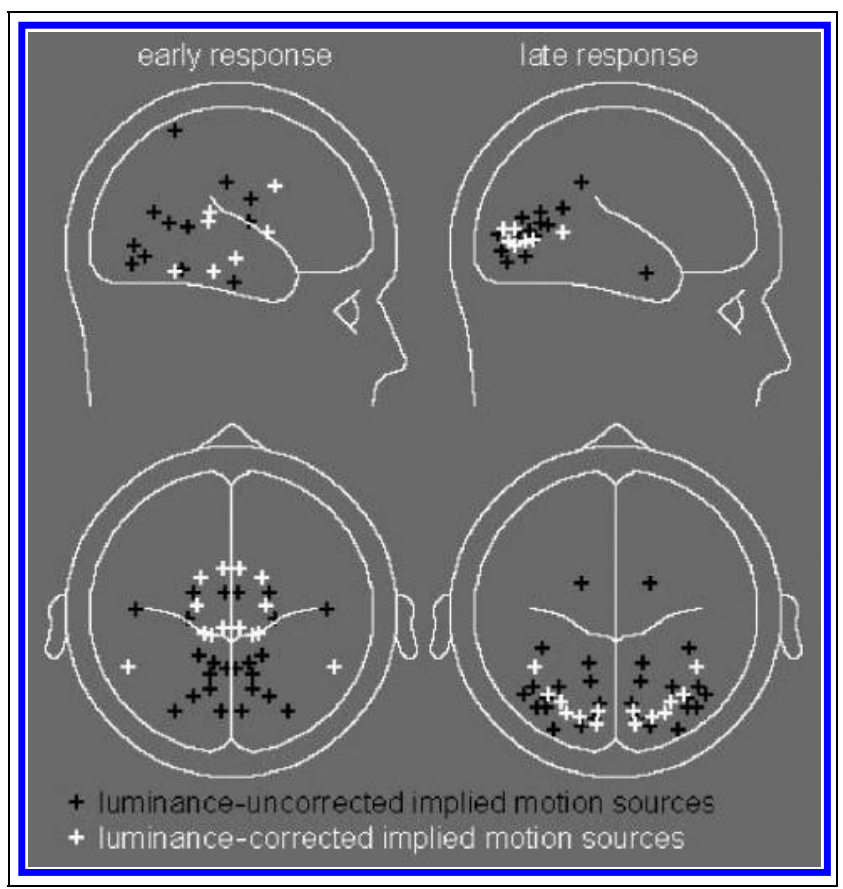

Figure 4. Individual source models for the early implied motion response (60 to $100 \mathrm{msec}$ in luminance-uncorrected, and 120 to $160 \mathrm{msec}$ in luminance-corrected experiment; left) and for the late response (260 to $320 \mathrm{msec}$ in luminance-uncorrected, and 280-400 msec for luminance-corrected experiment; right). model cannot account for; the lower the RV, the better the model) ranged between $3.5 \%$ and $40.2 \%$ (mean \pm SEM $14.9 \% \pm 2.8 \%)$. For two participants, the BESA model revealed dipole pairs outside the brain. These sources were excluded from further analysis regarding early implied versus nonimplied source positions. Because the BESA models of these participants were within limits for all other conditions, the two participants were not excluded from other dipole analysis.

In contrast, a BESA model for the implied motion response (260 to $320 \mathrm{msec}$ ), which contained one symmetric dipole pair in the occipital lobe, resulted in a model scalp distribution that was for $98.3 \%$ equal to the scalp distribution in the grand-average data. The location of this source was in concordance with an extrastriate source, possibly hMT + . The model was then refitted per participant $(n=14)$ to obtain individual sources (Figure 4). RVs of those individual models ranged between $3.4 \%$ and $25.9 \%$ (mean \pm SEM $10.9 \% \pm 1.8 \%)$. Individual sources of the late implied motion effect were located closely together, whereas the early sources were much more widespread.

\section{Luminance-corrected Source Analysis}

The late implied motion response (300 to $400 \mathrm{msec}$ ) in the luminance-corrected experiment could also be nicely fitted by a single dipole pair model. BESA analysis of the grand-average data resulted in a dipole model very similar to that of the luminance-uncorrected data. The model was again tested on the individual data of the seven participants (mean RV \pm SEM 16.7\% $\pm 4.0 \%$ ) and refitted. RVs for the individual sources ranged between $1.8 \%$ and $12.1 \%$ (mean \pm SEM $7.5 \% \pm 1.4 \%$ ). Individual sources (Figure 4) overlapped with the luminanceuncorrected implied motion sources. Three one-way ANOVAs along three axes revealed that the source locations of both groups did not differ significantly ( $p$ values were $.63, .32$, and .49). We thus conclude that luminance does not affect this late implied motion response.

Individual sources of the early difference (120 to $160 \mathrm{msec}$ ) in response to luminance-corrected implied and nonimplied photographs were established (mean \pm SEM RV $7.5 \% \pm 0.9 \%$ ). The luminance-corrected sources were located more anterior than the uncorrected sources. Indeed, a one-way ANOVA along the posterior/anterior axis revealed that this difference bordered on significance with a $p$ value of .05 . The source locations did not differ significantly ( $p$ values were .89 and .62) along the other two axes.

\section{Inanimate RDP Motion}

\section{Visually Evoked Potentials}

Differences in evoked potentials between the moving, static, and flickering RDPs were clearly visible at the 
same occipital-parietal electrodes that revealed the implied motion response (Figure 5). The difference in VEPs between coherently moving and flickering RDPs was significant from $120 \mathrm{msec}$ until the end of the stimulus presentation at $500 \mathrm{msec}$, whereas the response to a moving and a static RDP differed significantly from 100 to $160 \mathrm{msec}$ and from 180 to $260 \mathrm{msec}$ (repeatedmeasures general linear model; Greenhouse-Geisser $p$ value $<.05)$.

\section{Source Analysis}

Source analysis of the motion versus static and of the motion versus noise differences revealed activity of several different sources. To isolate a motion source that could have been responsible for the implied motion response, individual (late) implied motion sources were fitted on the motion data. Best fits were found at an average latency of $155 \mathrm{msec}( \pm 7.5$ SEM). This latency overlaps with the 150 to 200 msec occurrence of the negative peak that was considered to be the directionselective hMT + response in previous studies (Hoffmann et al., 2001; Probst et al., 1993). RVs of the implied models on the motion data were, however, still high (39.9\% \pm 4.8 SEM for motion vs. static, and $40.2 \% \pm$ 2.9 SEM for the motion vs. noise condition), meaning that either implied and real motion do not share the same source, or that other motion sources are also active during the same period.

Next, the individual sources were refitted to obtain the optimal motion sources per individual (Figure 6). Real motion sources covered a much larger area than the implied motion sources, overlapping the location of the latter. This confirms that several motion sources were simultaneously active, which could explain the poor fit of the implied model. The location of the individual implied motion sources was compared with their corresponding motion-static and motion-flicker

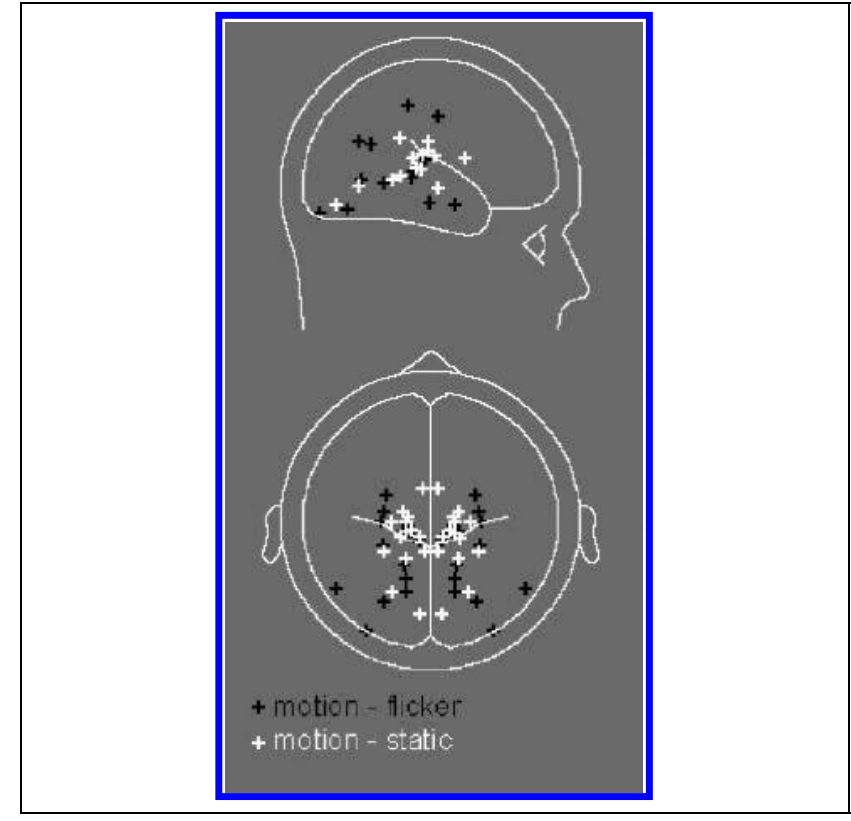

Figure 6. Neuronal sources for motion responses, as established by subtraction of motion versus flicker (black crosses) and motion versus static (white crosses) VEPs, are widespread across participants. They, however, do overlap with implied motion sources (which were shown in Figure 3).

sources along three axes in a multivariate test. The implied motion and motion-flicker sources did not differ significantly in a multivariate test (Hotelling's trace $p$ value $=.092$ ). However, implied motion and motion-static sources did differ significantly in a multivariate test (Hotelling's trace $p$ value $=.001$ ). Univariate analysis revealed that the implied motion sources were significantly more posterior and lateral than the motion-static sources. Apparently, implied motion activates a very specific part of the human motion analysis complex.
Figure 5. Grand average $(n=14)$ of evoked potentials to moving, static, and flickering (noise) RDPs differ maximally around 150 to $220 \mathrm{msec}$. The thick dotted lines and bars represent the difference curves and corresponding significantly differing periods of moving versus static and moving versus flickering RDPs. Differences are stronger at electrode PO4 (left) than at electrode $\mathrm{Pz}$ (right).

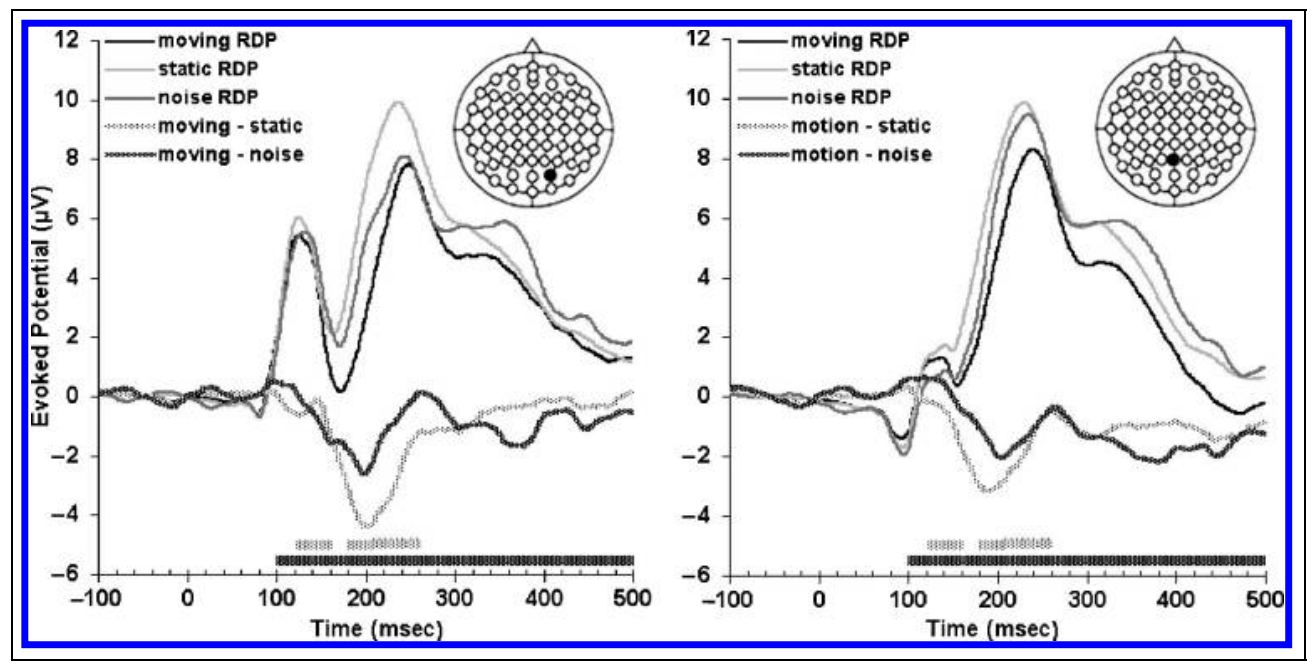




\section{Animate Motion}

\section{Visually Evoked Potentials}

To possibly obtain a more accurate localization of the sources of the VEP to real animate motion, we additionally recorded in a separate experiment the response to movie clips that depicted a human agent running from left to right or vice versa. This experiment was conducted in combination with the luminance-corrected experiment, which was described earlier, on seven subjects. The movie clip consisted of nine frames that were each presented for $70 \mathrm{msec}$. The onset of the responses to the movie clips (Figure 7) were delayed with about 60 msec compared to the onset of the inanimate RDP motion responses. This difference, however, could be attributed to the stimulus presentation. Motion was visible only after the second frame was presented. In the experiment with the moving RDPs, the second frame was shown after $12 \mathrm{msec}$, whereas for the movie clip the second frame was shown after $70 \mathrm{msec}$. Additionally, these participants had an overall longer response latency as was visible in the response to the luminancecorrected photographs (Figure 3), which may also account for this delay.

Differences in potential between implied motion photographs and movie clips were tested for significance with a repeated-measures general linear model. Responses differed significantly from 160 to 180; from 220 to 260; and from 320 to $360 \mathrm{msec}$ (GreenhouseGeisser $p$ value $<.05$ ).

\section{Animate Motion Source Localization}

As with inanimate RDP motion, the individual implied motion dipole sources were fitted on the difference between the movie-clip response and the response to the (luminance corrected) implied motion photographs (without subtraction of the response to the nonimplied photographs). As movie clips and implied photographs were similar in appearance and presence of implied motion, the difference between the two reflected a pure motion response. The implied sources fitted the data best around $260 \mathrm{msec}$ (mean latency = $259.1 \pm 9.2$ msec SEM, RVs ranged from $7.0 \%$ to $61.8 \%$; mean \pm SEM $26.7 \% \pm 7.3 \%$ ). After subtraction of the 60 -msec difference, this motion response is roughly in the same range as we report for the RDPs and as has been reported by others (Hoffmann et al., 2001; Probst et al., 1993).

Again we used a BESA model to estimate the source location of the animate motion sources. Individual animate motion sources are shown in Figure 8. Implied and animate motion sources were located closely together. The locations of the individual sources were compared along three axes in a multivariate test. This showed that implied motion sources and animate motion sources did not differ significantly in location (Hotelling's trace $p$ value $=.857$ ).

\section{Conclusions}

Based on response latencies and source analysis of both experiments, we conclude that implied motion evokes a delayed response in an area that overlaps with motionsensitive cortex (hMT+).

\section{DISCUSSION}

\section{Early versus Late Implied Motion Evoked Potentials}

Potentials evoked by photographs of human agents implying motion differed significantly from potentials evoked by photographs of the same human agents without implied motion in two periods: an early period (60 to 100 msec after stimulus onset in the first experiment and 120 to $160 \mathrm{msec}$ in the second experiment), and a late period (280 to $320 \mathrm{msec}$ in the first, and 300 to $400 \mathrm{msec}$ in the second experiment). The early response was much smaller in amplitude than the latter and was barely visible in the grand-average VEPs. Furthermore, the timing of the first deviation coincided with VEP
Figure 7. Grand average $(n=7)$ of evoked potentials to implied motion photographs (luminance-corrected) and to movie clips of a running person. The dotted line depicts the difference curve and the bars indicate time periods during which significant differences between the two conditions occur at electrode PO4 (left) and electrode $\mathrm{Pz}$ (right)

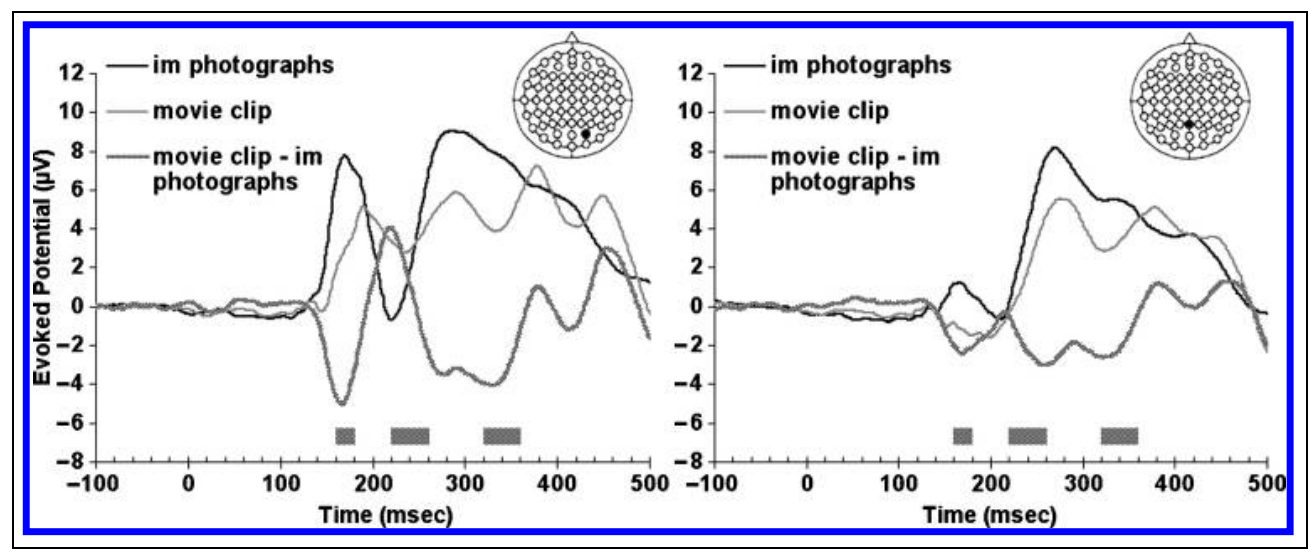




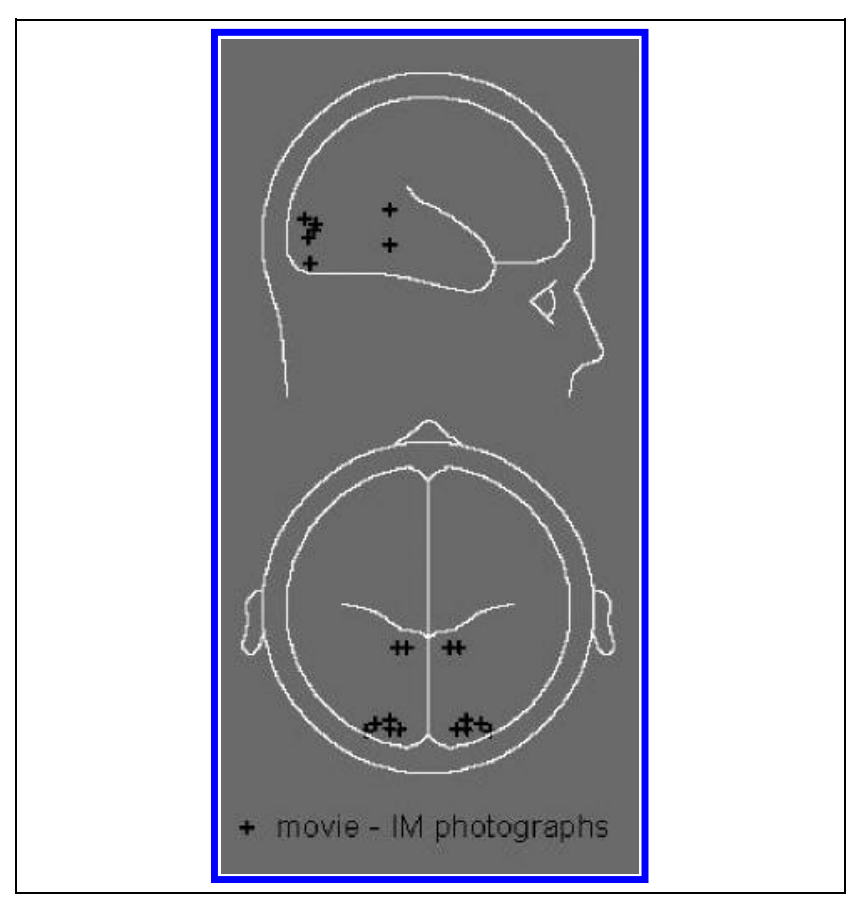

Figure 8. Individual source activity to animate motion was located in the occipital lobe for five out of seven subjects. This area overlapped with the late implied motion sources as shown in Figure 3.

responses to luminance (Johannes et al., 1995), spatial frequency (Kenemans et al., 2000), orientation (Arakawa et al., 2000), and size and eccentricity (Busch et al., 2004). Therefore, we reason that this early response was due to low-level stimulus differences between implied and nonimplied photographs.

BESA source locations for this early difference were established in both luminance corrected and uncorrected conditions. The luminance-corrected sources were more anterior than the uncorrected sources. This indicates that luminance is indeed largely responsible for this early response, but other low-level visual features may still play a role.

In contrast, the late difference in response to implied versus nonimplied photographs, which occurred from 260 to 400 msec after stimulus onset, cannot be explained by these low-level image differences as they occur earlier. Although Johannes et al. (1995) did find an interaction of luminance and attention from 350 to 750 msec after stimulus onset, this should not affect the responses in the current experiment, as the attention task of the participants was the same for both implied as nonimplied photographs. Furthermore, we showed that adjusting the luminance of the implied motion photographs to match that of the nonimplied photographs did not change the implied motion response. In both luminance-corrected and -uncorrected experiments, this late difference seemed to arise from a specific area in the occipital region. This indicates that the late difference is mainly caused by the presence versus absence of implied motion in the photographs, and we refer to this difference as the implied motion response.

\section{Delayed Response Latency of Implied Motion Compared to Real Motion}

The moving versus stationary RDPs were expected to evoke the largest difference in activity in area hMT + . However, earlier visual areas also show a large difference in response to these stimuli (Sunaert et al., 1999; Qian \& Andersen, 1994). Therefore, it might be difficult to extract the hMT + response from just this comparison. Because hMT + also responds well to flicker or random noise, the difference in hMT + activity induced by motion versus flicker might be expected to be smaller than by motion versus static. Furthermore, because lower visual areas as V1 discriminate less between motion and flicker (Sunaert et al., 1999; Qian \& Andersen, 1994), the relative contribution of hMT + to the subtracted evoked potential should be higher. Therefore, to optimally locate the motion-sensitive areas, both static and flickering RDPs were compared to moving RDPs.

Differences between moving and static RDP evoked potentials, and between moving and flicker RDP evoked potentials started at respectively 100 and $120 \mathrm{msec}$ after stimulus onset. It has been argued that the initial motion response around $120 \mathrm{msec}$ does not reflect hMT+ activity, as it is not susceptible to direction-selective motion adaptation. Instead, the negative peak around 170 to $200 \mathrm{msec}$ is described as a direction-selective motion response (Hoffman et al., 2001), which can be localized at the hMT + position (Probst et al., 1993). The "motion versus static" and the "motion versus flicker" difference curves in the present study also show this negative peak. Comparison of this peak with the implied motion response from 260 to 320 msec latency in the first experiment shows that the activation by implied motion is delayed with roughly $90 \mathrm{msec}$ compared to real motion activation. Furthermore, source-modeling shows that when fitting the locations of the individual implied motion sources on the individual RDP motion data in both experiments, these sources give their best fit around $155 \mathrm{msec}$. This indicates that the area involved in implied motion processing is involved in RDP motion processing at $155 \mathrm{msec}$ after stimulus onset, $105 \mathrm{msec}$ earlier than the implied motion response.

When fitting individual implied motion sources on their corresponding animate movie-clip data, they fit best around $260 \mathrm{msec}$. However, to make the speed of the runner in the movie clip as natural as possible, the frame rate in the clip was set to $70 \mathrm{msec}$. When subtracting the second frame presentation delay of roughly $60 \mathrm{msec}$ (compared to the \pm 10 -msec frame rate of the first experiment and other studies) from this latency, it approaches the RDP motion response of the first experiment and of other studies (Hoffmann et al., 2001; Probst et al., 1993) at 150 to $200 \mathrm{msec}$. Additionally, 
the implied motion response for the subjects that participated in this part of the experiments was also delayed, meaning that these subjects had an overall longer latency. The reason for this overall longer latency remains unclear. However, regardless of the overall latencies, the implied motion response for these subjects was delayed by $100 \mathrm{msec}$ compared to the response to animate motion $(260-60=200 \mathrm{msec}$ vs. $300 \mathrm{msec}$ after stimulus onset).

Thus, in both experiments, the implied motion response is delayed by approximately $100 \mathrm{msec}$ compared to real motion responses.

\section{Comparison of Neuronal Sources for Implied and Real Motion}

Dipole models to explain the RDP motion response showed a large variation in locations. This is not surprising, as several areas besides hMT + are responsive to motion (e.g., V1 and V3A; Sunaert et al., 1999). However, the area that contains the real motion sources overlaps with the area that is activated by implied motion. Comparison of the walking movie clip versus implied motion stills revealed well-localized motion sources in the occipital lobe. Furthermore, these sources were located in the same area as the individual implied motion sources. This indicates that implied motion evoked a response from an area in the motion-sensitive cortex.

The individual motion sources obtained from the comparison between the different RDP responses were less constant in location than the motion sources obtained from the movie clip of a walking agent versus an isolated frame from this clip. A possible explanation is that the RDPs (which covered the entire screen area) evoked a response in many areas, including those areas that were only just sensitive to coherent and/or incoherent motion or spatial change. On the other hand, the walker, which covered only a small part of the screen, might have evoked a response only in those areas that were highly sensitive to coherent motion. Neurons in such an area should have receptive fields that are large enough to cover the shift from one walk position to another. Because hMT + cells have such receptive fields, hMT + could thus be the motion-sensitive area found in this study.

\section{Feedback from Temporal Object Recognition Areas onto Motion Processing Areas}

This study showed that the neural response to implied motion in human motion areas is slower compared to the response to real motion, which suggests that the implied motion information arrives at this area via a different, longer pathway. This "feedback" might well arise from the superior temporal sulcus (STS) region, which seems to be specialized for the perception of bodily actions and postures in both macaque monkeys (Jellema \& Perrett, 2002) and humans (Allison, Puce, \& McCarthy, 2000). Of particular interest for the current study is that cells in the anterior part of the macaque STS respond to specific articulated body movements and postures, whether executed by an actor or expected to happen on the basis of the immediately preceding perceptual history (Jellema \& Perrett, 2003a), or when implied by the articulation of limbs in a static body posture (Jellema \& Perrett, 2003b). This view is in agreement with Senior et al., 2000, who, besides the response in $\mathrm{hMT}+$, reported a difference in BOLD response for the implied versus nonimplied motion conditions in temporal regions, and with Kourtzi and Kanwisher (2000), who showed additional implied motion activation in the STS.

The current EEG study, however, does not clearly demonstrate activity in temporal regions following implied motion. The early response from 60 to $100 \mathrm{msec}$ seems too early for STS activity, and individual sources were not consistently found at temporal locations. Because the STS is thought to discriminate between implied and nonimplied responses, it contains cells responsive to either or both conditions. The difference in response to both conditions might thus be smaller than the difference in response in hMT + . Furthermore, the response from the STS might overlap in time with responses related to stimulus onset and low-level stimulus differences in other areas, which might obscure the STS response. This might explain why the temporal activation did show up in both fMRI studies, which can easily differentiate between responses from different areas, but not in the spatially poorer EEG.

\section{Role of Form Feedback onto Motion Processing Areas}

Basically, the processing of visual form and motion is thought to occur along two different neural pathways: motion along the dorsal pathway, and form along the ventral pathway (Ungerleider \& Mishkin, 1982). However, in an observer's percept, the two are combined. Feedback processes as described in the current study may contribute to this combined percept. Implied motion may activate posture-dependent neurons in the temporal cortex, which in turn may activate hMT+ neurons that are sensitive to motion in the same direction as was implied. This activation would enhance the percept of that particular motion. Thus, implied motion would cause the static object to "jump out" from other static objects that do not possess implied motion cues. At the same time, it would "prepare" the observing agent for the type and direction of motion that is most likely to happen next. This would be particularly advantageous when the observer itself is in motion or making eye movements, or when detecting an animate object in 
an inanimate moving surrounding, for instance, a rabbit in rustling, tall grass. Further, studies on representational momentum have shown that implied motion distorts an observers' memory for the location of an object in the direction of the implied motion (Freyd, 1983). Thus, implied motion may help to predict the motion path and future position of an object.

\section{METHODS}

\section{Participants}

Ten men and nine women participated in the first experiment (luminance-uncorrected implied motion stimuli and RDPs). Five subjects had an excessive number of artifacts in their data and were therefore excluded from VEP analysis, leaving data from seven men and seven women. All were right-handed, had normal or corrected-to-normal visual acuity, and were aged between 20 and 31 years. Ten of the participants were naive to the purpose of the study, two women (both authors) and two men were not. Naive and informed subjects only differed significantly in early components of the RDP responses. Because there was no significant difference between the two subject groups in the main condition of this experiment (with vs. without implied motion), no distinction was made between naive and informed participants during analysis.

Three males and four female students participated in the second experiment (luminance-corrected photographs and animate motion movie clip). All participants were naive to the purpose of the study, right-handed, with normal or corrected-to-normal visual acuity, and aged between 21 and 32 years.

Naive participants in the first experiment were paid expenses, whereas subjects in the second experiment participated as part of their course work. All participants had given their written informed consent. Experimental procedures were conducted in concordance with the Declaration of Helsinki (World Medical Association 2000).

\section{Experiment 1: Stimuli and Task}

Stimuli were presented within a round aperture (radius of $3.3^{\circ}$, black surround) on a 17-in. monitor (1024 by 780 pixels, $84 \mathrm{~Hz}$ ) at a viewing distance of $1 \mathrm{~m}$. In the first experiment, two types of gray-scale pictures were used: photographs and RDPs. Photographs showed one of three different human agents either running towards the left or right, or standing still at left or right profile view, against a gray background (edited with Photoshop). Persons in the photographs were $6^{\circ}$ in height. The RDPs had been made by scrambling photographs (with and without implied motion) into dots of 5 by 5 pixels. During the experiment, the RDPs could be moving coherently in the left or right direction at ap- proximately $6.8^{\circ} / \mathrm{sec}$, they could be stationary, or could be presented in random order as flicker (at $84 \mathrm{~Hz}$ ).

Stimuli were presented for $500 \mathrm{msec}$ followed by an interstimulus period of $1 \mathrm{sec}$ (black screen). To ensure that subjects attended to all stimuli, for both the photograph and RDP conditions, a target-recognition task was included. When an RDP with dots twice the size of the stimulus dots was presented, participants had to press a button. This RDP could be moving, stationary, or flickering with the same parameters as the test RDPs. Also, when a photograph of a person from the frontal angle (either running or standing) was presented, subjects had to press the button. Ten percent of all stimuli were targets for these tasks. All subjects detected well above $90 \%$ of the control stimuli. The number of false alarms ranged between 0 and 18. No subjects were excluded based on their task performance. Throughout the whole recording session, a red fixation dot was present in the middle of the screen.

\section{Experiment 2: Stimuli and Task}

Experiment 2 contained the same gray-scale photographs as used in the first experiment, except that the luminance of the persons (not the background) in the implied photos had been altered to match that of their nonimplied counterparts. Original luminance of the three nonimplied and implied pairs in Experiment 1 was respectively 22.3 versus $23.7,21.2$ versus 20.5 , and 23.2 versus $23.3 \mathrm{~cd} / \mathrm{m}^{2}$, and has been altered to 22.3 , 21.7 , and $23.2 \mathrm{~cd} / \mathrm{m}^{2}$.

The animate motion movie clip consisted of nine frames depicting the same three human agents running from one side of the aperture to the other. Every frame was presented for $70 \mathrm{msec}$. At this frame rate, the speed of the running person was roughly $9^{\circ} / \mathrm{sec}$, which seemed to match the speed expressed in the limb movements in the clip. Stimuli were presented for $630 \mathrm{msec}$ with a 1000-msec interstimulus period. Participants were again requested to push a button when the person in the photographs (not in the movie clips) was standing or running forwards.

\section{Procedure}

Participants were seated in a comfortable chair in a darkened room. All participants received the same instructions regarding the task, and examples of the test stimuli and task stimuli were given prior to the experiment. All were requested to fixate on the red dot on the screen and to make eyeblinks only in the interstimulus periods. Both experiments were run in Presentation (Neurobehavioral Systems, Albany, CA).

In Experiment 1, RDPs, photographs, and task stimuli were presented 720 times in random order, in six 15-min blocks. Due to fatigue, two subjects were unable to complete more than $67 \%$ and $90 \%$ of the trials, respectively. 
Because their task performance and the percentage of rejected artifacts were within limits, data of these subjects were included in the final analysis.

In Experiment 2, movie clips, photographs, and task stimuli were presented 400 times in random order, in four 17-min blocks. No subjects needed to be excluded on the basis of their behavioral data or number of artifacts.

\section{Apparatus and Recording}

The EEG was recorded from $59 \mathrm{AgCl}$ scalp electrodes. Additional electrodes included one grounding electrode, one electrode on each mastoid, two electrodes above and below the left eye for vertical EOG, and one electrode next to each eye for horizontal EOG. Resistance between skin and electrodes was kept below $5 \mathrm{k} \Omega$ throughout the experiment. BrainVision Recorder (Brain Products, Germany) was used to sample and digitize the EEG at $1 \mathrm{kHz}$, filter (high-pass cutoff at $0.03 \mathrm{~Hz}$, low-pass cutoff at $400 \mathrm{~Hz}$, and a 50-Hz notch filter), and store on hard disk for off-line analysis. Electrode $\mathrm{Cz}$ was used as reference during the experiment. All scalp electrodes, including $\mathrm{Cz}$, were off-line re-referenced to averaged mastoids for further analysis. For the BESA source analysis, all electrodes were re-referenced against the average scalp potential.

\section{Data Reduction and Analysis}

BrainVision Analyser was used for data analysis. The EEG was segmented into stimulus-locked epochs from $100 \mathrm{msec}$ before to $500 \mathrm{msec}$ after stimulus onset. Segments containing an attention-task stimulus or a (false) response in a test stimulus were removed from further analysis. Epochs including blinks, eye movements (criteria $\pm 60 \mu \mathrm{V})$, or artifacts $( \pm 120 \mu \mathrm{V}$ on any EEG channel) were discarded. Participants for which more than $50 \%$ of the segments had been excluded were discarded from further analysis (5 out of 19 participants). Lastly, VEPs were filtered (high-pass cutoff $0.05 \mathrm{~Hz}$, lowpass cutoff $20 \mathrm{~Hz}, 12 \mathrm{~dB} / \mathrm{oct}$ ) and baseline corrected for 100 to 0 msec before stimulus presentation.

For every participant, VEPs were segmented into 20 -msec fragments. The average potentials of these fragments were used to signify differences between responses to the two photograph conditions, between the moving and stationary RDPs, and between the moving and flickering RDPs. A general linear model with repeated measures was performed over all subjects, using condition and electrode location as withinsubjects factors, and naivety as between-subjects factor. Greenhouse-Geisser corrected $p$ values $<.05$ were considered significant.

Individual VEPs were used to calculate the grandaverage VEPs over all subjects. Additionally, the grandaverage VEP of photographs without implied motion was subtracted from the grand-average VEP of photographs with implied motion. Moving minus stationary, and moving minus flickering RDP grand averages were obtained similarly.

\section{Source Localization}

To locate the neuronal sources underlying the differences in evoked potentials, BESA 2.2 (Scherg \& Picton, 1991) was performed. This method modeled location, orientation, and strength of equivalent intracranial dipole sources according to the recorded scalp activity. The optimal dipole solution was found by searching for a minimum in the RV function. To reduce the probability of interacting dipoles (i.e., adjacent dipoles with opposing high-amplitude potential fields), the energy constraint of the BESA model was set to $20 \%$ (with the remaining $80 \%$ for the $\mathrm{RV}$ criterion), thus favoring source solutions with relatively low dipole strengths (Berg \& Scherg, 1994). Single dipole pairs were used for source models. The location and orientation of the dipoles were bilateral symmetrically constrained.

\section{Acknowledgments}

We thank Bert V. van den Berg and two anonymous reviewers for their helpful comments on the manuscript. This study was supported by The Helmholtz Research Institute, Utrecht University; the Dutch Science Organization (NWO); and the Belgian Science Policy IUAP program.

Reprint requests should be sent to Richard J. A. van Wezel, Department of Functional Neurobiology, Utrecht University, Padualaan 8, 3584CH Utrecht, The Netherlands, or via e-mail: R.J.A.vanWezel@bio.uu.nl.

\section{REFERENCES}

Allison, T., Puce, A., \& McCarthy, G. (2000). Social perception from visual cues: Role of the STS region. Trends in Cognitive Sciences, 4, 267-278.

Arakawa, K., Tobimatsu, S., Kurita-Tashima, S., Nakayama, M., Kira, J. I., \& Kato, M. (2000). Effects of stimulus orientation on spatial frequency function of the visual evoked potential. Experimental Brain Research, 131, 121-125.

Berg, P., \& Scherg, M. (1994). BESA version 2.0 bandbook. Munich: Megis.

Britten, K. H., Shadlen, M. N., Newsome, W. T., \& Movshon, J. A. (1992). The analysis of visual motion: A comparison of neuronal and psychophysical performance. Journal of Neuroscience, 12, 4745-4765.

Busch, N. A., Debener, S., Kranczioch, C., Engel, A. K., \& Herrmann, C. S. (2004). Size matters: Effects of stimulus size, duration and eccentricity on the visual gamma-band response. Clinical Neuropbysiology, 115, 1810-1820.

Freyd, J. J. (1983). The mental representation of movement when static stimuli are viewed. Perception \& Psychopbysics 33, 575-581.

Hoffmann, M. B., Unsold, A. S., \& Bach, M. (2001). Directional tuning of human motion adaptation as reflected by the motion VEP. Vision Research, 41, 2187-2194. 
Jellema, T., \& Perrett, D. I. (2002). Coding of visible and hidden objects. Common mechanisms in perception and action. Attention and Performance, 19, 356-380.

Jellema, T., \& Perrett, D. I. (2003a). Perceptual history influences neural responses to face and body postures. Journal of Cognitive Neuroscience, 15, 961-971.

Jellema, T., \& Perrett, D. I. (2003b). Cells in monkey STS responsive to articulated body motions and consequent static posture: A case of implied motion? Neuropsychologia. 41, 1728-1737.

Johannes, S., Münte, T. F., Heinze, H. J. \& Mangun, G. R. (1995). Luminance and spatial attention effects on early visual processing. Cognitive Brain Research, 2, 189-205.

Kenemans, J. L., Baas, J. M., Mangun, G. R., Lijffijt, M., \& Verbaten, M. N. (2000). On the processing of spatial frequencies as revealed by evoked-potential source modeling. Clinical Neurophysiology, 111, 1113-1123.

Kourtzi, Z., \& Kanwisher, N. (2000). Activation in human MT/MST by static images with implied motion. Iournal of Cognitive Neuroscience, 12, 48-55.

Krekelberg, B., Dannenberg, S., Hoffmann, K. P., Bremmer, F., \& Ross, J. (2003). Neural correlates of implied motion. Nature, 424, 674-677.

Probst, T., Plendl, H., Paulus, W., Wist, E. R., \& Scherg, M. (1993). Identification of the visual motion area (area V5) in the human brain by dipole source analysis. Experimental Brain Research, 93, 345-351.
Qian, N., \& Andersen, R. A. (1994). Transparent motion perception as detection of unbalanced motion signals: II. Physiology. Journal of Neuroscience, 14, 7367-7380.

Scherg, M., \& Picton, T. W. (1991). Separation and identification of event-related potential components by brain electric source analysis. Electroencephalography and Clinical Neurophysiology, Supplement, 42, 24-37.

Senior, C., Barnes, J., Giampietro, V., Simmons, A., Bullmore, E. T., Brammer, M., \& David, A. S. (2000). The functional neuroanatomy of implicit-motion perception or representational momentum. Current Biology, 10, 16-22.

Senior, C., Barnes, J., Giampietro, V., Simmons, A., Bullmore, E. T., Brammer, M., \& David, A. S. (2002). Representational momentum and the brain: An investigation into the functional necessity of V5/MT. Visual Cognition, 9, 81-92.

Sunaert, S., Van Hecke, P., Marchal, G., \& Orban, G. A. (1999). Motion-responsive regions of the human brain. Experimental Brain Research, 127, 355-370.

Tootell, R. B., Reppas, J. B., Kwong, K. K., Malach, R., Born, R. T., Brady, T. J., Rosen, B. R., \& Belliveau, J. W. (1995). Functional analysis of human MT and related visual cortical areas using magnetic resonance imaging. Journal of Neuroscience, 15, 3215-3230.

Ungerleider, L. G., \& Mishkin, M. (1982). Two cortical visual systems. In D. J. Ingle, M. A. Goodale, \& R. J. W. Mansfield (Eds.), Analysis of visual behavior (pp. 549-586). Cambridge: MIT Press. 


\section{This article has been cited by:}

1. Nobu Shirai, Tomoko Imura. 2014. Implied motion perception from a still image in infancy. Experimental Brain Research 232, 3079-3087. [CrossRef]

2. Stephen C. Hedger, Howard C. Nusbaum, Olivier Lescop, Pascal Wallisch, Berthold Hoeckner. 2013. Music can elicit a visual motion aftereffect. Attention, Perception, \& Psychophysics 75, 1039-1047. [CrossRef]

3. Kentaro Yamamoto, Kayo Miura. 2012. Time dilation caused by static images with implied motion. Experimental Brain Research 223, 311-319. [CrossRef]

4. Thomas D. Albright. 2012. On the Perception of Probable Things: Neural Substrates of Associative Memory, Imagery, and Perception. Neuron 74, 227-245. [CrossRef]

5. Preston P. Thakral, Lauren R. Moo, Scott D. Slotnick. 2012. A neural mechanism for aesthetic experience. NeuroReport 23, 310-313. [CrossRef]

6. Jeannette A. M. Lorteije, Nick E. Barraclough, Tjeerd Jellema, Mathijs Raemaekers, Jacob Duijnhouwer, Dengke Xiao, Mike W. Oram, Martin J. M. Lankheet, David I. Perrett, Richard J. A. van Wezel. 2011. Implied Motion Activation in Cortical Area MT Can Be Explained by Visual Low-level Features. Journal of Cognitive Neuroscience 23:6, 1533-1548. [Abstract] [Full Text] [PDF] [PDF Plus]

7. Andrea S. Piotrowski, Lorna S. Jakobson. 2011. Representational momentum in older adults. Brain and Cognition . [CrossRef]

8. Alessandro Moscatelli, Laura Polito, Francesco Lacquaniti. 2011. Time perception of action photographs is more precise than that of still photographs. Experimental Brain Research . [CrossRef]

9. Andrea Pavan, Luigi F. Cuturi, Marcello Maniglia, Clara Casco, Gianluca Campana. 2011. Implied motion from static photographs influences the perceived position of stationary objects. Vision Research 51, 187-194. [CrossRef]

10. Kenneth F. Valyear, Jody C. Culham. 2010. Observing Learned Object-specific Functional Grasps Preferentially Activates the Ventral Stream. Journal of Cognitive Neuroscience 22:5, 970-984. [Abstract] [Full Text] [PDF] [PDF Plus]

11. Nicole M. Taylor, Lorna S. Jakobson. 2010. Representational momentum in children born preterm and at term. Brain and Cognition 72, 464-471. [CrossRef]

12. Marina A. Pavlova, Matthias Wecker, Kerstin Krombholz, Arseny A. Sokolov. 2010. Perception of intentions and actions: Gender stereotype susceptibility. Brain Research 1311, 81-85. [CrossRef]

13. Tjeerd Jellema, Jeannette Lorteije, Sophie van Rijn, Mascha van t' Wout, Edward de Haan, Herman van Engeland, Chantal Kemner. 2009. Involuntary interpretation of social cues is compromised in autism spectrum disorders. Autism Research 2:10.1002/ aur.v2:4, 192-204. [CrossRef]

14. M PAVLOVA. 2009. Perception and understanding of intentions and actions: Does gender matter?. Neuroscience Letters 449, 133-136. [CrossRef]

15. Zoe Kourtzi, Bart Krekelberg, Richard J.A. van Wezel. 2008. Linking form and motion in the primate brain. Trends in Cognitive Sciences 12, 230-236. [CrossRef]

16. Jeannette A. M. Lorteije, Richard J. A. van Wezel, Maarten J. van der Smagt. 2008. Disentangling neural structures for processing of high- and low-speed visual motion. European Journal of Neuroscience 27, 2341-2353. [CrossRef]

17. Marina Pavlova, Alexander N. Sokolov, Niels Birbaumer, Ingeborg Krägeloh-Mann. 2008. Perception and Understanding of Others' Actions and Brain Connectivity. Journal of Cognitive Neuroscience 20:3, 494-504. [Abstract] [PDF] [PDF Plus]

18. Jonathan Winawer, Alexander C. Huk, Lera Boroditsky. 2008. A Motion Aftereffect From Still Photographs Depicting Motion. Psychological Science 19, 276-283. [CrossRef]

19. J. L. ALFORD, P. VAN DONKELAAR, P. DASSONVILLE, R. T. MARROCCO. 2007. Transcranial magnetic stimulation over MT/MST fails to impair judgments of implied motion. Cognitive, Affective, \& Behavioral Neuroscience 7, 225-232. [CrossRef]

20. Jeannette A. M. Lorteije, J. Leon Kenemans, Tjeerd Jellema, Rob H. J. van der Lubbe, Marjolein W. Lommers, Richard J. A. van Wezel. 2007. Adaptation to Real Motion Reveals Direction-selective Interactions between Real and Implied Motion Processing. Journal of Cognitive Neuroscience 19:8, 1231-1240. [Abstract] [PDF] [PDF Plus]

21. Sven P. Heinrich. 2007. A primer on motion visual evoked potentials. Documenta Ophthalmologica 114, 83-105. [CrossRef]

22. Nick E. Barraclough, Dengke Xiao, Mike W. Oram, David I. PerrettThe sensitivity of primate STS neurons to walking sequences and to the degree of articulation in static images 135-148. [CrossRef] 